\title{
Uso Racional de Medicamentos no Contexto da Estratégia de Saúde da Família: só acesso não basta!
}

\author{
Rational Drug Use in the Context of the Family \\ Health Strategy: only access is not enough!
}

Maurício de ASSIS*, Juliana LEAL Universidade Estadual de Feira de Santana, Feira de Santana-BA, Brasil. Av. Transnordestina, S/N, Bairro Novo Horizonte CEP 44036-900 Feira de Santana-BA, Brasil.E-mail: msdeassis@outlook.com

\section{ABSTRACT}

According World Health Organization, the Rational Drug Use (RDU) requires the patients receive the appropriate medicine for their clinical condition, in the proper dose for their needs, for an adequate period of time, by the lowest cost to them. Other important requirement for RDU is the patients should handle the medicine according to it specifications and they should follow the usage guidelines communicated by health professionals. This study aim to describe the knowledge of the users of a Family Health Unit (FHU), concerning medicines and drugs, according to the information related to RDU. The work was carried out by a descriptive cross-sectional study. Data collection was conducted through a form, applied to a random sample of patients of FHU $(\mathrm{N}=18)$, between February and March 2012. Considering the user's knowledge about the drug name, correct identification of the packaging of the prescribed medicine, doses and proper storage, it was understood that the level of knowledge of the studied population is partial; therefore, insufficient for the RDU. It was observed that patients are not aware of essential information concerning their own medicines. Even though they receiving the appropriate medicine for their own clinical condition, meaning suitable dose, correct period required correct treatment the majority of patients could not identify their own medicines or handle and properly use them. Also, URD requires the provision, to patients, of adequate information about the pharmacotherapy; because only the access to medicines, even those indicated for patients clinical condition is not enough.

KEYWORDS: Primary Health Care; Drug Utilization; Knowledge; Pharmacotherapy.

\section{RESUMO}

A Organização Mundial de Saúde afirma que para ocorrer Uso Racional de Medicamentos (URM) a pessoa usuária deve receber o medicamento apropriado para sua condição clínica, em dose adequada para suas necessidades, por um período de tempo suficiente, ao menor custo. Outro requisito importante é que a pessoa usuária precisa manusear o medicamento conforme suas especificidades e seguir as orientações de uso transmitidas pelos profissionais de saúde. Este estudo objetivou descrever o conhecimento dos usuários de uma Unidade de Saúde da Família (USF) sobre medicamentos, em função das informações relacionadas ao URM. Trata-se de um estudo de delineamento transversal descritivo, a coleta de dados foi realizada através de um formulário, aplicado a uma amostra aleatória dos usuários da farmácia de uma USF ( $\mathrm{N}=18)$, entre fevereiro e março de 2012. Considerando o conhecimento do usuário acerca do nome do medicamento, identificação da embalagem do medicamento a ser utilizado, aspectos posológicos e de armazenamento, entendeu-se que o nível de conhecimento da população estuda é parcial e insuficiente para o URM, tendo em vista que os mesmos desconhecem informações essenciais. Os entrevistados recebiam o medicamento apropriado para a sua condição clínica, com dose também adequada, para utilizar no período necessário ao tratamento, sem despesa financeira e ainda assim a maioria dos entrevistados não sabia identificá-los tão pouco manusear e utilizar adequadamente. Deve-se atrelar ao URM a adequada prestação de informações acerca da farmacoterapia, o acesso do usuário ao medicamento indicado para sua condição clínica não significa necessariamente uso racional, não basta.

PALAVRAS CHAVE: Atenção Primária a Saúde; Uso de Medicamentos; Conhecimento; Farmacoterapia. 


\section{LISTA DE ABREVIAÇÕES:}

URM - Uso Racional de Medicamentos

USF - Unidade de Saúde da Família

SINITOX - Sistema Nacional de

nformações Tóxico-Farmacológicas

DCB - Denominação Comum Brasileira

AF - Atenção Farmacêutica

\section{INTRODUÇÃO}

Tido o medicamento como objeto da saúde, percebe-se sua importância para o indivíduo sadio e enfermo, no sentido de proporcionar qualidade de vida por meio da promoção, proteção e recuperação da saúde. Considera-se que para ocorrer o Uso Racional dos Medicamentos (URM) a pessoa usuária deve receber o medicamento apropriado para sua condição clínica, em dose adequada para suas necessidades individuais, por um período de tempo suficiente e ao menor custo para si e para a comunidade (1).

No entanto, quando utilizados ou manuseados indevidamente, os medicamentos também podem causar danos a saúde. Nestas situações, perde o usuário ao ter sua saúde prejudicada por esse insumo, seja pela ação do fármaco no organismo ou pela omissão do medicamento em combater um agravo. Perde também o Estado, tendo em vista que o enfermo pode ser entendido como sinônimo de custo para o sistema de saúde. Perde ainda a sociedade, uma vez que o individuo deixará de contribuir para com a mesma por afastar-se da execução de suas atividades cotidianas para cuidar de sua saúde.

Vivenciamos um cenário em que mais da metade dos medicamentos são prescritos, dispensados ou utilizados de forma inadequada (1). Sendo a falta de informação sobre os aspectos do tratamento uma das principais razões para que 30 a $50 \%$ das pessoas usuárias não utilizem os medicamentos conforme foram prescritos (2).

No Brasil, dados do Sistema Nacional de Informações Toxico-Farmacológicas - SINITOX ratificam as estatísticas, mostrando que os medicamentos ocupam a primeira colocação dentre os agentes causadores de intoxicação humana no país (3). Estes dados enfatizam o potencial nocivo que os fármacos podem adotar, bem como a importância do URM para o estado denominado saúde.

Um estudo publicado em 2011 acerca da organização da Assistência Farmacêutica na Estratégia de Saúde da Família de um município baiano, afirma que existe uma prática de dispensação que não promove uma contribuição ao usuário do medicamento, pois é desprovida de cuidados como a avaliação da prescrição, orientações quanto ao uso do medicamento, no sentido de verificar se o usuário compreendeu claramente as informações sobre o medicamento que está usando, a indicação, a duração do tratamento, as doses, os possíveis efeitos colaterais, como preparar, quando parar, entre outros aspectos pertinentes ao uso de medicamentos (4).

O presente estudo trata do uso de medicamentos em uma Unidade de Saúde da Família, cenário onde muitas vezes a figura do farmacêutico é ausente, ou seja, tem o potencial em indicar pontos a serem melhorados no que diz respeito a etapa de dispensação de medicamentos, mais especificamente ao processo de orientação aos usuários quanto ao uso e manuseio de medicamentos. Nesta perspectiva, este estudo apresenta como objetivo descrever o nível de conhecimento sobre medicamentos pelos usuários de uma USF, de Feira de Santana-BA, por meio da investigação do nível de conhecimento em três eixos: apresentação farmacêutica utilizada, aspectos posológicos e armazenamento domiciliar dos medicamentos.

\section{MATERIAIS E MÉTODOS}

Trata-se de um estudo de delineamento transversal de natureza descritiva. O campo de estudo consistiu da USF de um dos distritos da cidade de Feira de Santana-BA.

A coleta de dados foi realizada por meio de um formulário, aplicado nos meses de fevereiro a março de 2012, após a devida aprovação no Comitê de Ética em Pesquisa, aos usuários hipertensos e diabéticos que se dirigiram a USF para adquirir medicamentos (18 usuários). Este grupo foi escolhido tendo em vista que os protocolos de tratamento da hipertensão e diabetes requerem uso contínuo de medicamentos, logo estão mais expostos aos aspectos inerentes ao uso destes produtos.

As entrevistas foram realizadas na farmácia da USF logo após a entrega do medicamento ao usuário. Assim, o pesquisador poderia requisitar a cópia da prescrição e o medicamento dispensado, objetos que auxiliaram o preenchimento do formulário. Registra-se que a prescrição emitida pela USF é digitalizada, logo sua leitura não era dificultada por questões de legibilidade.

Para coleta dos dados, foi requerida dos usuários que relatassem o nome do fármaco utilizado assim como sua dosagem, a quantidade da forma farmacêutica utilizada a cada administração, o intervalo de tempo entre as doses e a via de administração, foi requerido ainda que os mesmos descrevessem o local onde armazenavam os medicamentos. Para avaliar o armazenamento domiciliar dos medicamentos consideraram-se as especificações do Formulário Terapêutico Nacional de 2010.

Aos usuários que utilizavam mais de um fármaco indagou-se qual embalagem pertencia cada medicamen- 
to, isto porque os medicamentos eram dispensados em sua embalagem primária. Durante a entrevista os entrevistados podiam consultar a prescrição e a própria embalagem do medicamento.

Como parâmetro para descrever o nível de conhecimento dos entrevistados nos diferentes eixos pesquisados, adotou-se os termos:

a) "conhecem", para os entrevistados cientes das informações;

b) "conhecem parcialmente", para os entrevistados que não estavam totalmente cientes das informações e;

c) “desconhecem”, para os entrevistados que ignoraram quaisquer informações.

Os gráficos, bem como seus dados estatísticos foram gerados com o auxílio do programa Microsoft Excel 2010.

Aspectos Éticos: Este estudo foi realizado em conformidade com a Resolução do Conselho Nacional de Saúde número 196, de 10 de outubro de 1996 - Diretrizes e Normas Regulamentadoras de Pesquisa Envolvendo Seres Humanos, sendo registrado no Comitê de Ética em Pesquisa da Universidade Estadual de Feira de Santana sob protocolo número 078/2011 e com numeração do Certificado de Apresentação para Apreciação Ética equivalente a 0082.0.059.000-11.

\section{RESULTADOS E DISCUSSÃO}

\section{Eixo: Conhecimento da Apresentação Farmacêutica}

Investigou-se a capacidade das pessoas usuárias em relatar o nome dos medicamentos que utilizava, seja pela Denominação Comum Brasileira (DCB), seja pela nomenclatura fantasia. Os resultados estão mostrados na Figura 1.

Figura 1. Conhecimento dos usuários atendidos pela farmácia de uma Unidade de Saúde da Família de Feira de Santana, Ba, quanto ao nome do fármaco que utiliza.

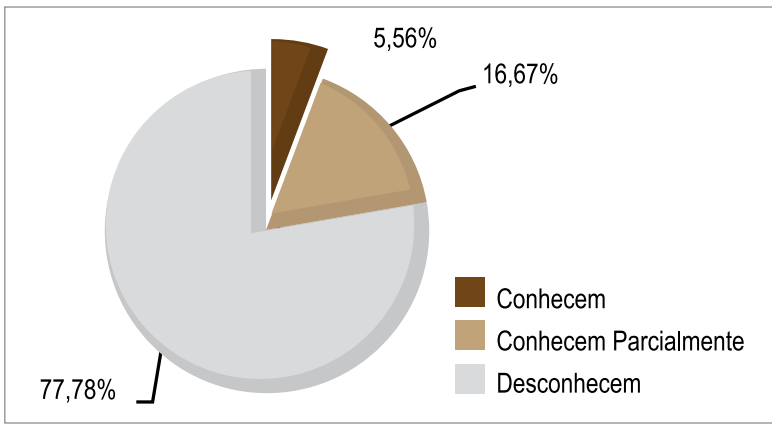

Pela análise da Figura 1, constata-se que 94,44\% dos entrevistados apresentavam dificuldades em conhe- cer o essencial ao utilizar um medicamento, que é saber o seu nome. Os mesmos $94,44 \%$ dos entrevistados não souberam afirmar qual a dosagem do(s) medicamento(s) prescrito(s). Todos os sujeitos da pesquisa, que souberam informar o nome do fármaco utilizado, o fizeram por meio do emprego da DCB.

Dados de outros levantamentos acerca da temática divergem dos resultados encontrados por este estudo. A literatura descreve que a maioria dos usuários conhece o nome do medicamento utilizado, bem como sua dose $(2,5)$. No entanto, estes levantamentos foram realizados em ambiente urbano, enquanto que a população desse estudo foi predominantemente rural. Dessa forma, pode-se apontar então que as características sócio-demográficas dos dois ambientes contribuíram para a divergência encontrada entre o nível de conhecimento das respectivas populações.

Independente de qual seja o fármaco que uma pessoa faça uso, é imprescindível que se conheça o seu nome, tendo em vista que existem situações nas quais é necessário identificá-lo. É importante lembrar que para a realização de determinados exames laboratoriais, administração de medicamentos em casos de urgência ou emergência, pesquisa de reações de hipersensibilidade, e até mesmo para a prescrição de outros medicamentos necessita-se saber se a pessoa atendida faz uso de algum outro fármaco.

É essencial que na polifarmácia o usuário possa identificar a embalagem de cada apresentação farmacêutica a fim de evitar trocas no momento da administração do medicamento. Entre os entrevistados que utilizavam mais de uma apresentação farmacêutica $(94,44 \%)$, investigou-se a capacidade destes em distinguir a embalagem a qual pertencia cada medicamento, os achados são apresentados na Tabela 1 .

Tabela 1: Conhecimento dos usuários atendidos pela farmácia de uma Unidade de Saúde da Família de Feira de Santana, Ba, quanto à embalagem do medicamento utilizado.

\begin{tabular}{|c|c|}
\hline USUÁRIOS & PORCENTAGEM \\
\hline Conhecem & $38,89 \%$ \\
\hline Conhecem Parcialmente & $27,78 \%$ \\
\hline Desconhecem & $33,33 \%$ \\
\hline
\end{tabular}

A análise da Tabela 1, mostra que $61,11 \%$ dos entrevistados apresentavam dificuldade em reconhecer a embalagem do medicamento a ser utilizado, o que é preocupante, pois existe risco da pessoa usuária administrar doses do medicamento não prescritas e/ou prejudiciais em virtude de utilizá-los em horário inadequado.

Na farmácia da USF os medicamentos são dispen- 
sados em sua embalagem primária, fato que contribui para que a pessoa usuária tenha problemas para identificar o medicamento. Por exemplo, o blister de diferentes apresentações farmacêuticas de mesmo laboratório compartilham muitas características comuns de layout, tornando muitas vezes a distinção entre as embalagens possível apenas com minuciosa leitura, o que influi significativamente para os resultados encontrados. Soma-se a isso, o tamanho das letras impressas nos blisteres que em muitos casos é registrada em tamanhos minúsculos dificultando a leitura das pessoas com baixo nível de escolaridade ou acometidos por problemas ópticos.

\section{Eixo: Aspectos Posológicos}

O cumprimento da posologia é um fator essencial ao bom uso do medicamento, tendo em vista que é a posologia que determina qual a concentração ideal do fármaco no organismo do usuário, garantindo uma biodisponibilidade adequada a ação farmacológica sem o aparecimento de reações toxicológicas. Assim, quando o paciente não segue as recomendações de administração dos medicamentos acaba por interferir na eficácia, efetividade e segurança do medicamento, uma vez que a concentração plasmática desejada do fármaco é comprometida.

Os fatores limitantes para o cumprimento da posologia, encontrados por este estudo, centraram-se no desconhecimento dos usuários quanto à utilização do medicamento. Por exemplo, pacientes afirmaram não utilizar o medicamento no horário indicado por não conhecer o intervalo de tempo que deveria ser dado entre uma dose e outra. $\mathrm{O}$ mesmo pode ser dito quanto à quantidade da forma farmacêutica a ser administrada por dose. O conhecimento dos entrevistados quanto aos aspectos posológicos (dose, via de administração e intervalo entre as doses) é mostrado na Figura 2. Esses dados refletem a adesão dos entrevistados à posologia indicada.

Figura 2: Conhecimento dos usuários atendidos pela farmácia de uma Unidade de Saúde da Família de Feira de Santana, Ba, quanto aos aspectos posológicos da medicação

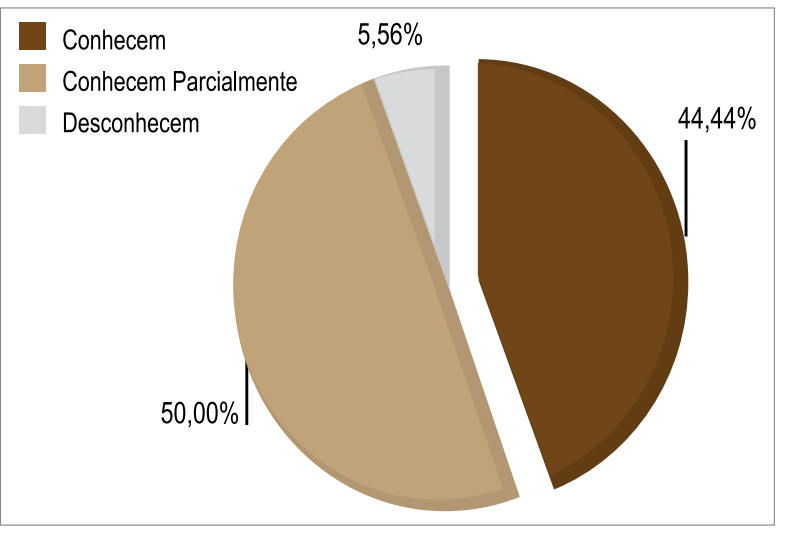

Utilizar determinado medicamento descumprindo as recomendações posológicas faz com que se perca o significado e o objetivo da terapia farmacológica, uma vez que o fármaco desenvolverá seu mecanismo de ação de modo pouco eficiente com comprometimento da segurança de seu usuário.

\section{Eixo: Armazenamento Domiciliar dos Medicamentos}

Fazer uso de um fármaco adequado à condição clínica, conhecendo sua apresentação, indicação, efeitos adversos, precauções de uso e interações, além de seguir todas as recomendações posológicas ainda não é suficiente para que a abordagem farmacológica seja eficaz. Todos esses procedimentos se tornam ineficientes caso a forma farmacêutica esteja deteriorada devido a condições de armazenamento que comprometam a estabilidade do medicamento. As informações coletadas a respeito do armazenamento domiciliar dos medicamentos estão descritas na Figura 3.

A maioria dos entrevistados $(77,77 \%)$ descreveu locais adequados para o armazenamento dos medicamentos, ou seja, esses usuários conseguem teoricamente manter a estabilidade da formulação dentro dos meios disponíveis em sua residência. Os outros 22,23\% afirmaram não guardaro medicamento no local mais apropriado da residência, citando banheiro e cozinha, além de locais próximos a fontes de calor como eletrodomésticos. Frisa-se que não foram aferidos parâmetros como temperatura e umidade do local onde os medicamentos eram armazenados; o entrevistado apenas descreveu o ambiente e este era confrontado com as recomendações do Formulário Terapêutico Nacional de 2010.

Figura 3. Conhecimento dos usuários atendidos pela farmácia de uma Unidade de Saúde da Família de Feira de Santana, Ba, quanto ao armazenamento domiciliar do medicamento.

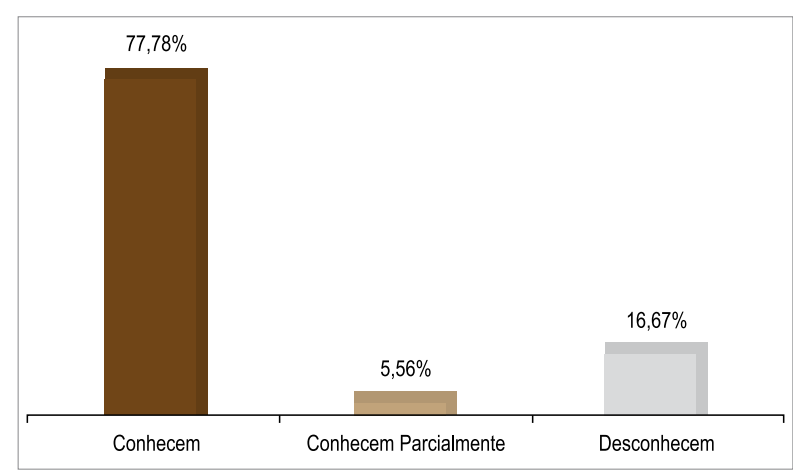

Eixo: Nível de Conhecimento dos Entrevistados Sobre Medicamentos e Seu Uso Racional

O presente estudo descreveu o nível de informação utilizando os termos "conhecem", “desconhecem" 
e "conhecem parcialmente", este último sendo utilizado nos casos onde foi investigada mais de uma informação em um único eixo (usuários que utilizavam mais de um medicamento, por exemplo), e o usuário não tenha demonstrado ter conhecimento de todas as informações sobre os medicamentos, mas que tenha mostrado conhecer ao menos uma. Em se tratando de uma determinada informação, considerou-se que ou o entrevistado a conhecia ou a desconhecia, não sendo utilizado um meio termo ("conhecem parcialmente"), tendo em vista que para que uma informação cumpra seu papel, deve ser entendida em sua totalidade, considerando as distorções que uma informação incompleta pode provocar.

Outros autores optam por classificar o conhecimento dos usuários utilizando os parâmetros: "bom nível de conhecimento", "nível regular de conhecimento" e "nível insuficiente". Como exemplo tem-se o trabalho de Martins et al (2012), no qual um estudo realizado em farmácia comunitária demonstrou que $57 \%$ de seus entrevistados possuíam um nível regular de informação, enquanto que $34 \%$ possuíam um nível de informação bom e $34 \%$ possuíam um nível insuficiente de informação (6).

Considerando os eixos utilizados por este estudo como parâmetros norteadores, entendeu-se que o nível de conhecimento da população estudada acerca dos medicamentos por elas utilizados é parcial e insuficiente para o Uso Racional de Medicamentos, tendo em vista que a maioria dos entrevistados desconheciam informações de uso e manuseio das respectivas apresentações farmacêuticas.

$\mathrm{O}$ número médio de medicamentos utilizados por pessoa foi de 2,33 medicamentos, o que, a priori, não é um empecilho aos usuários no que diz respeito ao co- nhecimento das informações sobre os medicamentos, tendo em vista que os mesmos não utilizavam muitos simultaneamente. Consequentemente a quantidade de informações a ser absorvida por eles seria menor. Dessa forma, não ocorre Uso Racional de Medicamentos entre a maioria dos usuários analisados, tendo em vista o desconhecimento e descumprimento de alguns parâmetros necessários para a utilização correta do medicamento.

Ressalta-se que, assim como não existe saúde parcial, também não existe Uso Racional de Medicamento parcial; para a utilização correta do medicamento, todos os parâmetros de uso e manuseio devem ser respeitados.

\section{CONCLUSÃO}

Foi verificada fragilidade no processo de orientação das pessoas assistidas pela USF, traduzida pela carência de informações verificada nos entrevistados e derivada da ausência da Atenção Farmacêutica (AF). A equipe de saúde da família não contempla o farmacêutico, de modo que essa ferramenta de promoção da saúde é omitida pela Estratégia de Saúde da Família ao instituir verdadeiras farmácias na Atenção Primária. Esta fragilidade não decorre de má conduta dos profissionais que atuam na USF, considerando que não se espera deles a execução da $\mathrm{AF}$, já que suas respectivas ações em prol da saúde incluem outras vertentes do cuidado.

O acesso da população aos medicamentos deve vir acompanhado de informações acerca do fármaco prescrito a fim de possibilitar o URM, e assim cumprir com os objetivos de promoção, proteção e recuperação da saúde. Assim, o acesso do usuário ao medicamento indicado para sua condição clínica não significa necessariamente uso racional, ou seja, não basta é o suficiente.

\section{REFERÊNCIAS}

1. OMS. Medicamentos: uso racional de los medicamentos. Organización Mundial de La Salud Disponível em: http://www.who.int/ mediacentre/ factsheets/fs338/es/

2. Dresch AP. Caracterização do Nível de Conhecimento sobre medicamentos prescritos e prevalência de automedicação por pacientes ambulatoriais odontológicos. [Dissertação]. Porto Alegre: Universidade Federal do Rio Grande do Sul, UFRGS. 2008.

3. BRASIL. Ministério da Saúde. Fundação Oswaldo Cruz. Sistema Nacional de Informações Tóxico Farmacológicas - SINITOX. Casos registrados de intoxicação e/ou envenenamento. [21abr.2011]. Disponível em: http://www.fiocruz.br/ sinitox_novo/cgi/cgilua.exe/sys/start.htm?sid=349
4. Alencar TOS, Nascimento MAA, Alencar BR. Assistência Farmacêutica no SUS: articulando sujeitos, saberes e práticas. Feira de Santana: UEFS, 2011.

5. Silva T, Schenkel EP, Mengue SS. Nível de informação a respeito de medicamentos prescritos a pacientes ambulatoriais de hospital universitário. Cad. Saúde Pública. 2000. 16(2):449-455.

6. Martins MS, Nascimento RM, Carvalho MN. Nível de Informação de Pacientes Hipertensos Atendidos em Farmácia Comunitária do Município de Belém, PA. INFARMA. 2012. 24(4/6):23-26. 